\title{
Galilei was wrong: Angular nonradial effects of radial gravity depend on density of matter
}

\author{
Jakub Czajko \\ Department of Science and Mathematics Education, Southern University and A \& M College, \\ Baton Rouge, LA 70813, USA \\ E-mail address: sunswing77@netscape.net
}

\begin{abstract}
Although in mathematical sense the actual meaning of Galilei's experiments conducted at leaning tower of Pisa is that he failed to detect any measurable impact of composition of matter on gravitating bodies, the obvious failure is mistakenly interpreted as experimental confirmation of lack of the impact instead. Galilei did not really perform internal validity checks of his experiments, because he did not ensure that he actually measured what he was supposed to measure. However, a modern experiment devised to test the impact of large mass on gravitational phenomena has revealed presence of (formerly unanticipated) extraneous frequency decrease in rays coming from Taurus A, when they passed close to our Sun (i.e. near occultation). The experiment has effectively confirmed that density of matter of the mass source of locally dominant gravitational field (which was our Sun's field) affects gravitational interactions happening on equipotential surfaces surrounding gravity center of the field. Also very similar experiment involving radio waves, which too exhibited (formerly unexpected) frequency decrease when they traveled along practically equipotential surface of Earth, has reaffirmed that conclusion. Hence contrary to Galilei, effects of nonradial (i.e. tangential and/or binormal) components of radial gravitational force fields depend (inversely) on (equipotential exposure to) the, assumed as practically constant and uniformly distributed, density of matter of the mass source of the local field.
\end{abstract}

Keywords: Potential energy; work done; nonradial gravitational effects; density of matter

\section{INTRODUCTION}

Physicists often argued that if a solid consisted entirely of stationary particles under no other forces than the attractive ones obeying the classic inverse square law of gravitation, then it could not be stable and thus would collapse to almost zero volume. Admission of a repulsive gravitational force could prevent that [1], provided the force would act in nonradial directions. Since apparent stability of most slowly evolving macroscopic solids is evident in common experiences on time scales of appreciable duration, then perhaps the idea is not implausible.

Repulsive force of gravity can also explain (at least a part of) the observed increasing redshift that is interpreted as accelerating expansion of the universe.

As a matter of fact, actual presence of certain nonradial effects of the usual radial gravitational field, which was revealed as formerly unanticipated frequency decrease of 
rays/waves passing near surfaces of our Sun and of Earth, confirmed existence of detectable intrinsic gravitational repulsion [2].

For just as increase in the rays' own energy $E$ (which is actually just another name for frequency $v$ of the rays/waves - due to the basic formula $E=h v$ where $h$ denotes the Planck constant) corresponds to an attractive force, sustained decrease of their frequency would correspond thus to definitely repulsive force - compare sketch in [3].

Notice that the path of a deflected ray passing near source mass of the locally dominant gravitational force field is also twisted while being curved by the field. Although ignored in former physics, the nonradial twist, which is mathematically unavoidable and thus indispensable in general [4], is formally quite equivalent to angular repulsion performed by the local field [5].

To grasp the idea, consider this example: In order for an airplane in flight to make a turn, it has to lean on the wing that points in the general direction of the attempted turn. The leaning, however, tilts also the airplane's tail with respect to the (assumed as being locally flat) surface of the Earth, so that even the (initially intended as planar) turn has an extra angular effect in the third, vertical dimension too.

Also a cyclist riding on fast moving bicycle must tilt it by leaning sidewise towards the incoming curve in order to stay on the road, unless the road is tilted and thus imposes a certain twisting tilt on the bicycle.

Either the rider must lean or the road must tilt, but the twist must be made in order to curve the bicycle's path for the coming turn, because - according to proven and thus unquestionably valid Frenet-Serret (F-S) formulas of differential geometry - all curvilinear motions are screwlike [4]. As every rigorously proven operational formula of mathematics, the F-S formulas are not just optional suggestions. They are the operational laws that we should always obey, especially in operational definitions of notions/concepts.

When performed by the gravitational force field itself, however, the twisting means that an extra work must be done by the field at the expense of potential energy of the field - see [4]. Since the extra nonradial twist was routinely ignored in former (i.e. created prior to 2000 $\mathrm{AD})$ physics and mathematics, the potential energy that is spent on the nonradial angular twist was never really accounted for, and thus the nonradial effects due to the nonradial angular twist were commonly deemed as "unanticipated".

Consequently thus, all the other than purely radial experiments, in which the (previously unrecognized) extra nonradial angular twist manifested its presence, were left unexplained and their data unreconciled [2].

Why then was the extra nonradial angular twisting effect unanticipated in the former physics, where work done and the corresponding to it potential energy is meticulously calculated, whereas the necessity to accommodate the extra twist has already been recognized and applied by most engineers designing roads and cars, whose knowledge relies not only on experience, but is rooted in physics too?

This was so because rate of work done by force fields (and thus also potential energy) was incompletely defined in former mathematics and physics. Incomplete operations are always detrimental to precise calculations, and can be especially harmful for drawing generalized conclusions from incompletely defined notions. 


\section{FORMER DEFINITION OF WORK DONE RATE AND POTENTIAL ENERGY IN RADIAL/CENTRAL FORCE FIELDS WAS INCOMPLETE}

Formerly, the generic rate $\mathrm{dW}$ at which work done $\mathrm{W}$ increases (or decreases, for that matter) has been mathematically misdefined as $\mathrm{dW}:=\mathbf{F} \cdot \mathrm{d} \mathbf{r}$ i.e. as the inner (scalar) product of vectors of force $\mathbf{F}$ and distance rate dr. The former definition clearly violates the absolutely mandatory product differentiation rule (the proof of which is uncontested) [6], according to which the rate ought to be defined as total differential, namely as $\mathrm{dW}(\mathbf{F} \cdot \mathbf{r})=(\mathbf{F} \cdot \mathrm{d} \mathbf{r})+(\mathbf{r} \cdot \mathrm{d} \mathbf{F})$ in order for it to be lawful as well as conceptually accurate. The formerly ignored right-handside (RHS) term $\mathbf{r} \cdot \mathrm{d} \mathbf{F}$ has evidently nonradial character [6], because the radial distance $r=|\mathbf{r}|$ is fixed for this particular nonradial operation and therefore only the force vector $\mathbf{F}$ actually varies therein. The radial distance $r$ and turning angle of the radius $\mathbf{r}$ can be assigned any valid values, but once assigned these values remain unchanged in the RHS term.

The former definition really pertains only to purely radial rate of work done, not to its total rate. Since potential energy is not streaming in open space like a river whose flow is usually confined within its banks but most likely spreads like unconstrained flood, the former incomplete formula not only understated the total amount of spent potential energy, but misrepresented the character of its flow too.

Although omission of inconsequential terms is admissible in approximations, exclusion of operationally necessary expressions in definitions is not. Definitions are conventional, but not really arbitrary. Thus definitional conventions should be lawful and operationally complete. Definitions must not be allowed to violate any laws of mathematics, for this might lead to veiled misconceptions. Disrespect for their own laws resulted in previous inability of sciences to explain the nonradial effects that were discarded in the former, operationally incomplete definition [2].

Notice that the fixed distance $r$ plays the role of just a constant parameter in the nonradial term, determining the particular equipotential surface that surrounds the gravity center of the locally dominant radial/center-bound gravitational force field. Only variables appearing under differentials are actually actively varying.

Pretty common linguistic misconception in mathematics is that all so-called variables do vary. Only variables denoting functions which appear in differentials actually do vary in strict mathematical sense of the term 'varying'. But symbols appearing apart from differentials (even though they are called variables) should rather be called unknowns instead, for they are either parameters (whose value is to be assigned) or functionals, i.e. values to be calculated yet. Once a particular value is assigned to a parameter or functional, the value remains fixed for the duration of the operation pertinent to the situation modeled by the operation.

Once assigned a value, parameters/functionals remain the same, fixed for the particular situation for which they had been determined. Thus being designated as 'functional' is just temporary situational attribute, which can be changed from functional to function and vice versa. The transition of the symbol's status from functional to function or vice versa is not quite arbitrary, however. Only symbols representing actually varying functions could be meaningfully differentiated and thus only they can signify functions. Parameters and functionals must never be differentiated, because they represent just assigned fixed values not meant to vary.

Hence in order to avoid ambiguity, if a variable $\mathrm{v}$, for example, is tentatively designated as a proper function in a certain situation yet it appears apart from any differentials, I shall write it as representing function $\mathrm{v}()$ to distinguish it from its functional/parameter denoted by just $\mathrm{v}$. The very same symbol can play the role of an active function of some independent 
variables in one operation, as well as that of a fixed functional/parameter in another situation, depending on need of each particular situation. The conceptual distinction between actively varying functions and their fixed functionals is essential for understanding synthetic reasonings in mathematical and physical sciences - it is thus conceptually imperative.

\section{IMPLICATION OF OPERATIONALLY COMPLETE DEFINITION OF WORK DONE AND POTENTIAL ENERGY OF RADIAL FORCE FIELDS}

At this classical depth of inquiry into gravitational phenomena I assume that magnitude of the radial force vector of gravity is given by Newton as $\mathrm{F}=-\mathrm{GMm} / \mathrm{r}^{2}$. Here the test mass $\mathrm{m}$ is assumed as being insignificantly small so that its own contribution to the strength of the local force field can be neglected. If these two interacting masses $\mathrm{M}$ and $\mathrm{m}$ were comparable in size and were located reasonably close to each other, then their respective force fields could be combined into a single amalgamated field, as if due to single mass source. However, there is no meaningful way to amalgamate two distinct gravitational force fields which are generated by two distant mass sources, whose gravity centers are located far apart. For amalgamating distant source masses could introduce force vectors that may not be radial with respect to gravity center of at least one of these source masses.

Since presence of nonradial effects of the usual radial/center-bound (central) force fields is unavoidable in general [4], from the above operationally complete (and thus mathematically lawful) definition of work done rate, the following new, operationally correct, comprehensive geometric formula has been derived in [6]:

$$
\begin{gathered}
\mathrm{dW}(\mathbf{F} \cdot \mathbf{r}):=\mathrm{d}(\mathbf{F} \cdot \mathbf{r}) \equiv(\mathbf{F} \cdot \mathrm{d} \mathbf{r})+(\mathbf{r} \cdot \mathrm{d} \mathbf{F})= \\
=-\mathrm{F} \cos 2 \alpha \mathrm{dr}+2 \mathrm{Frsin} 2 \alpha \mathrm{d} \alpha-\mathrm{rcos} 2 \alpha \mathrm{dF}
\end{gathered}
$$

where the equipotential term $2 \mathrm{Fr} \sin 2 \alpha \mathrm{d} \alpha$ depends on originally planar, varying spherical angle $\alpha$, i.e. the angle of visibility of the trajectory path pointed to by the pointing vector $\mathbf{r}$ whose value $r=|\mathbf{r}|$ denotes distance between gravity centers of two gravitating masses $\mathrm{M}$ and $\mathrm{m}$. Here $\cos \alpha=r_{p} / r$ where $r_{p}$ is radius of perihelion (or perigee, in the case of Earth), and $F=$ $|\mathbf{F}|$ denotes magnitude of the usual, radial attractive force vector of Newtonian gravity of the radial/center-bound force field generated by its main source mass $M$ (henceforth assumed as locally dominant) [6]. Note that the equipotential (angular nonradial) term is repulsive for its sign is opposite to that of the usual radial term -Fcos $2 \alpha \mathrm{dr}$ which corresponds to attractive force, as well as to the linear nonradial term -ros $2 \alpha \mathrm{dF}$ which is also attractive.

Note that in former physics, theories of gravitation considered only the usual radial/central impact of the mass source of the field. Even if written in mutually orthogonal terms like the rectangular coordinates $\mathrm{x}, \mathrm{y}, \mathrm{z}$, they nonetheless meant merely decomposition of the radial force vector at the point of impact (i.e. where the test mass $m$ is located), not really any structural composition of the effective resultant force vector from the radial and possible nonradial subcomponents.

When a mathematical formula incidentally came up also with some other than purely radial components of force vectors or potentials, all those nonradial components were routinely disregarded [7] as if they were nonexistent/impossible or always inconsequential. When local gravitational field contains two or more distinct mass sources, however, the resultant/effective force field vectors could comprise also subcomponents that are not necessarily radial at the given point of impact, but may have some nonzero tangential and/or 
binormal components as well [4]. For it is not really the resultant (i.e. combined) force field vector that is actually acting, but in fact all its orthogonal/perpendicular (i.e. their directional or dimensional) subcomponents do act quite independently of each other [8].

At the present depth of inquiry (which - for the sake of simplicity - presumes that both these masses $\mathrm{M}$ and $\mathrm{m}$ are constant while the work is being done by the local force field) only motion-independent (i.e. quasi-static) effects are taken into account, because nominally massless rays/waves cannot be reasonably compared to the huge mass of our Sun or Earth. In fact, all parameters characterizing the radial/center-bound local force field are presumed to be constant for the purpose of this presentation. By quasi-static effects I mean that although such effects are motion-independent, their prerequisite is a certain necessary transport that has to move the test mass/particle $\mathrm{m}$ along some path on an equipotential surface, where magnitude of generic radial potential $1 / \mathrm{r}$ does not change. But the spherical angle that can be associated with radial potential can vary on equipotential surfaces and thus - as an actively varying variable - it should induce some nonradial effects.

Since differential means a certain rate of change, each independently varying variable that stands under differential induces thus a change of the whole function that is quite independent of all other changes induced by the other independently varying variables. This implies that just as varying radial variable does induce the usual (radial) general-relativistic gravitational frequency shift, varying nonradial angular variable must induce some nonradial angular frequency shift and varying nonradial linear variable must induce some nonradial linear frequency shift.

The term standing in the middle of the eq. (1) with the angular rate $\mathrm{d} \alpha$ (where the angle $\alpha$ varies along sections of equipotential surfaces) yields generic function of the rate of work done $\mathrm{w}_{\mathrm{rQ}} \mathrm{Q}(\mathrm{Q}(\lambda)$ ) for which the field's potential energy is spent along the equipotential surface (that surrounds the local gravity center) which is determined by the radius $r$ and codetermined by the constant matter density Q [5]:

$$
\mathrm{w}_{\mathrm{rQ}}(\mathrm{Q}(\lambda))=\frac{-4 \mathrm{kGMmr} \mathrm{p}^{2} \lambda}{3 \mathrm{Qr}^{4}}=: \mathrm{W}_{\mathrm{rr}_{\mathrm{p}} \mathrm{Q}}(\lambda)
$$

where the rate of work done depends on the function $\mathrm{Q}(\lambda)$ that yields exposure (of the test mass $m$ that traverses the locally dominant gravitational force field due to the source mass $M$ ) to the (assumed as uniformly distributed inside the surface and constant, for the purpose of this note) matter density $\mathrm{Q}$ of the source mass $\mathrm{M}$.

The exposure function $\mathrm{Q}(\lambda)$ depends primarily on how far the test mass $m$ travels along the equipotential surface and is thus codetermined by the distance $r$ between the bodies $M$ and $\mathrm{m}$, the distance $r_{p}$ to perihelion and constant density of matter $\mathrm{Q}$ of the big source mass $\mathrm{M}$. At the present depth of inquiry, the exposure function $\mathrm{Q}(\lambda)$ depends only on the spherical distance $\lambda$. It is codeteremined by $r$ but it does not depend on $r$ as it might be in a function $Q(r$, $\lambda$ ) of evolution of the mater density itself. At a deeper level of inquiry into gravitational effects I have already considered varying distribution of matter density within the source mass $\mathrm{M}$ [9]. The work done rate $\mathrm{w}_{\mathrm{rQ}}(\mathrm{Q}(\lambda)$ ) is function of (varying) spherical distance $\lambda$, but it is functional with respect to (fixed) distances $r, r_{p}$ and matter density $Q$. The masses, gravitational constant $\mathrm{G}$ and coefficient $\mathrm{k}$ also co- (or pre-) determine it.

The nonradial angular rate of work done in eq. (2) can be rewritten in terms relevant to much more generic, but perhaps easier to assess, physical situation: 


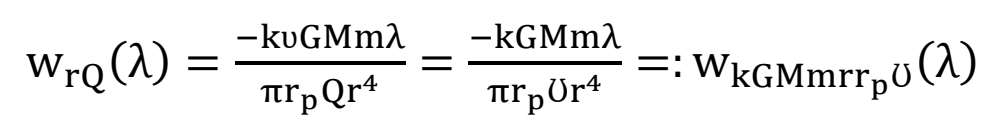

where $v$ is the perimeter volume enclosed by the fixed distance $r_{p}$ to the perihelion from the local gravity center of the mass $M$. Since the equipotential distance $\lambda=r \theta$ is codetermined also by the radial distance $r$ (which is limited from below by the radius of perihelion $\mathbf{r}_{\mathrm{p}}$ ) and by the spherical angle $\theta$ (which can be curvilinear, i.e. spheroidal spherical in general) that corresponds to the equipotential path $\lambda$.

Specific gravity is conventionally defined as $Q=Q_{M} / Q_{\text {water }}$ It is truly intensive (hence comparative) measure of density of matter of the source mass $M$ relative to density of water under normal conditions. The parameter $\delta=\mathrm{Q} / v$ yields the unit of relative density of the matter enclosed within the perimeter volume $v=4 \pi\left(r_{p}\right)^{3} / 3$. Hence assuming uniform density of matter and the perihelion being very close to surface of the source mass $M$, the potential energy of the local force field spent on the work done by the field depends (inversely though) on relative density of the matter contained inside the field, within the given radius of perihelion. In general thus, nonradial angular interactions happening along equipotential surfaces are codetermined by both dynamic and static characteristics of the local field: they are (virtually) directly proportional to mass density of the field's source mass $M$ and also inversely proportional to matter density Q of the field's source mass.

Neither Newton nor Einstein pondered gravitational interactions at this deep level of inquiry. Laws of physics are never final. They grow in complexity. Their form depends on their author's depth of inquiry into the relevant phenomena.

The RHS terms in the eq. (3) clearly indicate that relative density of matter of the source mass $\mathrm{M}$, which generates the local field, affects the rate at which work done by the force field is being compensated by potential energy of the field. This inference has already been confirmed by few experiments and many observations [2]. Although the eq. (3) is more comprehensive than the prior, experiment-driven formula that has been derived in [2], they are conceptually similar.

Since value of the radial potential always remains constant on equipotential surfaces surrounding the gravity center of the local gravitational force field, the potential energy expense taking place along such surfaces may be (conceptually) viewed as being quasi-static [10], for it has no explicit direct dependence on any parameters of motion of the two masses (such as their velocities or accelerations).

By Liouville theorem, in any integrable system with $\mathrm{n}$ degrees of freedom and $\mathrm{n}$ global constants one can always find local angular variables satisfying some conditions in addition to the regular action variables [11]. In the sense existence of angular nonradial effects of radial gravity is theoretically foreseeable, which conclusion can be reached also by other abstract mathematical reasonings [10].

However, purely mathematical investigations do not involve matter density. This is because pure mathematics was not really concerned with possible impact of composition (or any other physically measurable characteristics) of abstract geometric spaces, such as their density of mass or matter, for instance. While not unreasonable, the abstract number-points that conventionally fill up the abstract spaces explored by mathematicians do not really have size or any other physical attributes. Pure mathematics is thus inadequate for some physical applications.

This was the primary reason for developing the (new) synthetic mathematics that is supposed to take into account some very curious experimental hints, which suggested that even abstract mathematical spaces may possess certain physically distinguishable features 
implying thus possible presence of unknown yet abstract overarching pangeometric structures [12]. When a strict proof is not possible yet, synthesizing equations like the eq. (3) and then devising experiments to confirm the validity of predictions of such synthesized equations can be the best option to overcome the aforesaid conceptual deficiencies of current pure mathematics.

Abstractness is surely necessary for simplicity of generic reasonings, but the (commonly tolerated) abstracting away of even most essential defining features of both physical and abstract mathematical reality made pure mathematics not only inapplicable to some curious yet experimentally observed phenomena, but also confusing. For if pure mathematics does not really describe the physical reality that seemingly exists independently of our minds (whose presence is confirmed by unbiased experiments), whereas the artlike reality that it creates does not exist apart from the minds of its creators, then what is the purpose of their creation?

Modern mathematics became so enthralled with arbitrary reasonings that it tends to disregard hints supplied by curious experiments. Most of mathematical accomplishments are correct, but some were misguided and resulted in tacitly veiled misconceptions; the most severe ones relevant to this topic were discussed in [6]. The synthetic mathematics that I advocate is envisioned not just to rectify the misconceptions, but also to refocus mathematics on conceptual expansion. For although mathematics has developed elaborate methods for solving (ordinary and partial) differential equations, for example, no context-independent conceptual theory of inventing such equations exists apart from physics, which has its own unresolved issues - some with over three hundred years of lingering confusion.

Dependence of gravitational interactions on (exposure to) density of matter was not really anticipated by former physics, primarily because of the previous, operationally incomplete definition of work done and potential energy, but also because of traditional misinterpretation of meaning of the Galilei's experiments.

Both the present expression and the formerly obtained less detailed formula for nonradial angular (here equipotential) effects, which has already been derived from reasoning based upon curious but unbiased experimental hints [2], evidently challenge the traditional Galilei's conclusion that gravitational effects are not really affected by constitution of matter, which he has drawn from the famous experiments he conducted at the leaning tower of Pisa.

\section{GALILEI WAS WRONG: CONSTITUTION OF MATTER OF MASS SOURCES OF FORCE FIELDS AFFECTS NONRADIAL GRAVITATION}

Bondi has pointed out that although Galilei has found that all bodies fall (i.e. accelerate) equally fast at a given place (which fact has subsequently been tested also by Eötvös and then even more precisely by Dicke), there is a difference in their accelerations if they are in different places [13]. Since acceleration would disappear for freely falling observer, it is not really relevant observable - hence one should look at its variation with position and at the relative acceleration of neighboring particles, which - as tide-raising force - is universal observable, for it cannot be just removed or altered by any motion of the observer [13]. Recall that nonradial angular effects are acquired along equipotential surfaces surrounding local center of gravity, whereon magnitude of the radial/central potential remains unchanged so that the nonradial angular effects would appear as quasi-static [10].

When Galilei was dropping various objects from the leaning tower of Pisa, he observed that all of them reached the ground beneath in practically the same time. From that observation he has concluded that gravity is not affected by constitution (or density) of matter 
of those falling test bodies. Nonetheless, Galilei's evident failure to detect any perceptible impact of composition of matter on test bodies in free fall was traditionally hailed as confirmation of lack of the impact instead - an illogical twist that persists in virtually all presentations of the topic, despite known (even though formerly ignored) presence of experimental evidence to the contrary [2]. Lack of evidence does not constitute evidence of nonexistence.

However, Galilei's approach was based only on common sense, not on truly viable, exact reasoning. Galilei did not really perform mathematically satisfactory methodological analysis of his experiments. At that time it was not required to submit research proposal before conducting any experiments, of course. Before the time of Newton even the very notion of force of gravity was not (actionably) defined, certainly not in an operationally meaningful mathematical way.

Conclusions derived from more recent similar experiments were formulated much more cautiously, however, for Eötvös, for instance, simply stated that in a series of their experiments they could not detect any measurable deviation from the law of proportionality of inertial and gravitational mass [14].

But certain subsequent reinterpretations of their results - although insightful and factually quite correct - were far too general and therefore rather conceptually misleading [15]. Reanalysis of Eötvös-type experiments suggested indeed that their data are somewhat sensitive to composition of the materials used, but the (inferred) conjecture that the results would imply existence of intermediate-range coupling to baryon number or hypercharge was rather speculative [16] and not really absolutely necessary postulate [17].

Nonetheless, maybe a seed of truth, namely that composition of matter seems to matter (at least for some phenomena), was actually discovered by proponents of the "fifth" force. But they reanalyzed the Eötvös data only from the previous, mathematically incomplete radialonly paradigm of former physics. No absolutely correct inferences can be properly drawn from incompletely defined concepts. Yet that data surely hinted at the possibility that composition of matter does matter.

Only adequately designed and suitably conducted experiments could really establish or confirm facts (which are not always theory-neutral [18]), provided they are unbiased. Although the tentative theory postulating existence of the fifth force has been eventually dismissed, the long pending question of whether or not composition of matter does count was not really satisfactorily answered by the dismissal. Yet because numerical laws are established by experiments, evidently viable mathematical concepts are necessary to explain the numerical laws [19].

Nonetheless, because both justification and value of our concepts comes from their ability to predict and explain our experiences [20], we should also be able to operate on them in operationally complete and mathematically legitimate ways.

Let me recast the Galilei's observation in terms that are more adequate today: In gravitational force field of the Earth, small test bodies (external to the Earth) whose own force fields are disproportionately smaller than the Earth's field and thus are insignificant in the context of the Earth, kept on acquiring practically the same radial accelerations during their vertical free fall towards the Earth's gravity center. If stated this way, however, the same acceleration imparted on the falling test bodies might have been due to insufficiently precise observation or perhaps because of clear incomparability of those small test bodies' masses in presence of overwhelmingly large mass of the Earth, or due to both of these factors.

If one would rewrite the magnitude of the attractive Newtonian gravitational force in terms of abstract radial potentials of the classical gravitational force field due to the two 
masses $M$ and $m$, namely as $F=-G\left[\frac{M}{r}\right]\left[\frac{m}{r}\right]$ compare [12] - then it becomes clear that one would rather need to find at least two (definitely distinct and certainly unequal but preferably comparable) large masses $\mathrm{M}$ and drop the same test body (represented by the smaller mass $\mathrm{m}$ ) in each of these two different force fields generated by the two large masses, and then compare only impacts of the potentials of these large masses on the test mass $m$. Only in this hypothetical experiment such logically faulty (and thus definitely inadmissible in sciences) comparison of disproportionate masses (or of the potentials generated by their fields) could actually be avoided. The test mass is totally inconsequential there.

Obviously the only potential that really matters there is determined solely by the (presumed as huge) mass $\mathrm{M}$. The disproportionately small mass $\mathrm{m}$ is absent in the locally dominant, generic radial potential function $\left[\frac{\mathrm{M}}{\mathrm{r}}\right]$. If the test mass $\mathrm{m}$ is disproportionally small in comparison to the locally dominant mass $\mathrm{M}$, the radial potential created by the test mass is totally irrelevant there. It can serve only as a background, but it should never be used for comparisons (whether explicit or not) with the mass $M$ that is disproportionately larger than the test mass $\mathrm{m}$. One could not meaningfully compare differences in impact among those small test bodies either, because their masses (and potentials) are too small to discriminate among them in the contextual presence of the overwhelmingly large mass $\mathrm{M}$ of the Earth.

Since only potentials, not the forces they generate, are the truly fundamental building blocks of radial/center-bound force fields - as Aharonov-Bohm (A-B) effect indicated comparing forces actually means comparing just outcomes of interactions of the potentials, not the potentials themselves. This could be really confusing when some discrepant magnitudes of those potentials are essentially incomparable. The meaning of the A-B effect is that an impact of the potentials on charged particles exists even in the regions where the given fields (and thus also the forces acting on the particles) vanish - compare [21-23]. Since impacts of electromagnetic, electrostatic and gravitational forces are conceptually similar, the lesson learned from the A-B effect pertains to (and therefore it could be transferred onto) quasi-static gravitational fields. Seemingly, the force fields virtually emerge from pairing of potentials, which are the primary entities and therefore potentials evidently can manifest their actual presence even without the forces being actualized therein [12].

I am not faulting Galilei, but after Newton, and especially after Lagrange's grasp of the idea of generic radial potential (which he correctly envisioned as an abstract feature of the underlying radial/center-bound force field rather than as an attribute of a standalone single force), we no longer have excuse for professing that illogical Galilei's conclusion unchallenged.

The observed practically the same accelerations were not precise enough to draw his conclusion. One cannot infer reasonable deductions from observations in which some gravitational potentials due to those participating test masses could never be meaningfully compared with the potential of the Earth's field because of their disproportionate sizes. Therefore the prospective impact of composition of insignificantly small masses of the test bodies could never be precisely estimated even if carefully measured. Only the impact of composition of the huge mass $\mathrm{M}$ could be reasonably taken into consideration, but it must never be contrasted with that of those (too small to match the overwhelmingly big mass $\mathrm{M}$ ) test masses $\mathrm{m}$.

Hence yet another large mass (such as Sun or Moon) is really needed (for any reasonable comparison with the mass of the Earth and its impact) for hypothetical experiment like that of Galilei to yield any decisive inferences. Although Earth is significantly smaller in size and mass than our Sun, it is not disproportionately small. Hence mass of the Earth can be 
sensibly compared with mass of our Sun. Their masses and potentials are different but not unequivocally incomparable.

One should never attempt to compare essentially incomparable magnitudes because any inferences made from inadequate comparisons are prone to creating inadvertent misconceptions, even without misrepresentation of facts. Yet faulty virtual comparison is exactly what Galilei did while experimenting at the leaning tower of Pisa. His mishap needs to be exposed because sometimes it is still being replicated (and very similar, inadequate conclusions are still perpetuated); it still adversely affects even some modern theoretical reasonings and experiments.

Galilei has made great contributions to physical sciences of his time. There is no doubt about it. But he was unable to formulate methodology of his experiments in truly responsible manner, because the knowledge required for the alternative formulation I have offered above did not really exist before Newton published his Principia. Although Galilei did not make explicit mathematical comparisons of the test masses, his conclusion involved talking about those small test objects he dropped from the tower of Pisa, which is a kind of implicit or virtual comparison.

I am not criticizing Galilei. I just want the reader to understand what went wrong there. My reasoning is called conceptual analysis of operational terms in applied mathematics. It comes before designing experiments. To ensure fairly adequate (if not quite accurate) syntheses from experimental results, actual (or just implied) operations on all concepts involved therein should be both feasible and meaningful. The Galilei's common sense conclusion is clearly illogical and thus quite inadmissible. It is both operationally wrong and conceptually defective.

Since masses of those test bodies were plainly incomparable with the mass of the Earth, one would have to take just one such insignificantly small test mass $m$ and then throw it within gravitational force fields of several different and distinct planets and/or our Sun, whose masses can be compared with each other. This feat would eliminate the need to involve in comparisons the incomparably small force fields of those test bodies. Only then one could derive really reasonable inferences from comparisons of (the impact exerted by) each of the planets and/or the Sun.

Presumably taking the Galilei's conclusion at its face value, neither Newton nor Einstein (nor any other researcher) saw compelling reason to investigate also the possibility that - in other than purely radial interactions - density of matter could affect the nonradial (or just nonradial parts of mixed) interactions. For the possibility of existence of some other than radial interactions was mathematically foreseeable, because such interactions happen along the equipotential surfaces that enclose the source mass $\mathrm{M}$ of the locally dominant gravitational field [10].

For one to claim that an impact is beyond experimental means to detect it, if the impact could exist, is admissible. But to properly insist on nonexistence (as impossibility of existence) demands rigorous proof. One would have to actually prove that mathematically. Experiments are not substitutes for proofs - they can only supply hints suggesting existence but they could never prove nonexistence. The Galilei's claim could not be proved, however, for quite to the contrary, by the F-S formulas presence of nonradial angular effects is unavoidable in general [4].

It is then up to a theory to take such experimental hints and develop concepts upon which one could deduce predictions, which then may be confirmed (or not) by further experiments. Even if experiments confirm the predictions, this is not really existential proof, 
but only validation of viability of the theory that made the predictions, just verifying that the theoretical model behaves (but not necessarily works) just like the modeled phenomena.

Meanings of concepts can change in time so that they must be revisited and updated [24]. Yet the faulty Galilei's claim remained unchallenged for over three centuries while experiments contesting it remained quite unexplained up to $2000 \mathrm{AD}$ [2]. It stalled prospective development of some theories of mathematics and physics, whose designers did not dare to challenge the Galilei's dictum.

\section{HOW GALILEI-TYPE EXPERIMENTS SHOULD BE DEVISED}

If a test body of mass disproportionately smaller than that of the Earth could be dropped also on the Sun, for instance, one could compare both density of mass and of matter of the Earth with that of the Sun without the need to involve the test body in comparisons. One could then compare how the Earth and the Sun affect the very same kind of test body (even if it is disproportionally smaller than these celestial bodies) without ever trying to compare any of the celestial bodies with the test body or even the test bodies among themselves. Experiments to that effect have already been conducted and their results contradict the Galilei's claim that matter density does not count [2], even if it was not realized by their conductors.

However, a reader might point out that the eq. (3) pertains to effects acquired along equipotential surfaces, whereas Galilei experiments were purely radial. But dependency of the attractive pull of gravity on density of mass (which is related to density of matter at relative rest) has also been detected by several spacecrafts orbiting Moon. Recent experimental data obtained by the NASA's dual Gravity Recovery and Interior Laboratory (GRAIL) spacecraft have confirmed existence of free-air gravity anomalies due to mass concentrations (referred to as mascons) in lunar impact basins [25]. But the influence of mascons was considered only in terms of the usual radial effects of gravity field thus far, because - thanks to the faulty Galilei's claim - no theory of former physics ever bothered to study any nonradial angular effects of the usual radial/center-bound gravitational fields.

This particular result suggests that accounting for local fluctuations of density of mater is indeed necessary for more precise estimations of trajectories traversing radial gravitational force fields. In purely radial cases, however, the dependence is much more cumbersome to calculate, as it must involve also the data on crustal thickness of the Moon that is related to density of matter, which the present author has no access to. Hence (at some deeper than this current level of inquiry into gravitational interactions) both density of mass and density of matter of celestial bodies should be taken into account in investigations of both radial and nonradial gravitational effects, especially when they are very close to surface.

Nonradial effects should be included in estimations of radial fluctuations of effective gravity fields, because when only the radial effects are considered, then presence of just radial deformations of the field by some repulsive forces would merely diminish (or increase, depending on the extra radial forces' directions) the primary attractive force. This would be recorded as just an unspecified anomaly. If included, the nonradial effects could point to likely cause of the deformation.

By experimenting without adequate notions to express his thoughts, Galilei demonstrated courage bordering on exasperation with his fellow scientists' empty theoretical talks. But to present his intellectual desperation as a virtue of scientific approach to be imitated, seems like deification of ignorance. 
His experiment forfeited proper internal validity check, namely that he could really measure what he has intended (and supposed) to measure. Also by rushing to generalize his observations in absence of uncontestable methodology, he failed to ensure external validation of his experimental results too. Because of the two faults his research proposal would not have been approved by institutional review boards at most universities today. His deficient methodology should be exposed, but he should be praised as daring enthusiast of experimental physics, of course.

Nevertheless, it would be counterproductive to endorse experimentation as a substitute for operationally responsible thinking. His experiments should still be taught today, but perhaps just as examples of mathematically blind and thus rather uncritical, common sense thinking that is prone to tacitly veiled misconceptions.

For mathematically speaking, the common sense conclusion that Galilei has drawn from his experiments is a nonsense. If he had the hypothetical opportunity to drop the same ball also on the Sun, yet perhaps not exactly towards the gravity center of the Sun's field, his inferences (and following them general conclusions) might have been quite opposite. For validity of (an interpretation of) experimental results evidently depends also on the mathematical framework of the theory that instigated the experiment. Although mathematical framework is vital for proper reasonings, it is not a foundation that once established could last forever. It must be updated. Mathematical framework is always work in progress.

Beside internal and external validity checks, research proposals should ensure that all concepts to be used in experiments possess properly outlined domain of their validity, and that prospective operations to be performed on the concepts are not only feasible but also operationally complete and thus mathematically lawful. For operationally incomplete formulas are illegitimate and as such inadmissible.

The most general case is when test bodies would fall not exactly radially but partly also in certain nonradial (i.e. tangential and/or binormal) directions. Very analogous point of view had been offered also for electromagnetic fields - it was concisely discussed in [26]. Although even today it would be rather difficult to drop an object on the Sun or Moon, such a hypothetical experiment is actually feasible. In fact, Nature already does it for us.

For the Earth is continuously bombarded by streams of particles originating from deep space (including photons emitted by distant stars), some of which occasionally pass also near surfaces of the Sun or Moon or other planets, whose force fields' influence on energy of the photons passing by them could then be fairly precisely measured and compared once these particles arrive on Earth (and after their relativistic radial gravitational frequency shifts are taken into account).

As a matter of fact, some experimenters already took advantage of the great opportunity and intercepted particles coming from space, without realizing though that their experiments could contest the former (mathematically-adverse) common sense interpretation of those famous Galilei's experiments [2].

\section{EXPERIMENTS SHOWED THAT DENSITY OF MATTER COUNTS}

Sadeh has devised ingenious experiment akin to dropping a ball, which was passing by our Sun near its surface, and in another one the ball was passing by the Earth. The disproportionately small test balls were actually rays/waves. He has observed (unanticipated back then by theories of former physics [27]) extraneous frequency decrease in the rays coming from Taurus A when our Sun approached the rays' path on several consecutive days 
[28]. The closer the Sun approached the rays' line of sight, the deeper was their (deemed as extraneous) frequency decrease (redshift), which could not be explained, neither by plasma around Sun nor by general theory of relativity (GTR) [28]. The extraneous frequency decrease was practically almost exclusively tangential effect, because the purely radial gravitational frequency blueshift that was acquired on incoming part of the rays' trajectory path was cancelled out by the, also radial, gravitational frequency redshift that was acquired on the outgoing path of the Sun-centered (hence as if superposed) branch of the rays' decomposed path with respect to the center of our Sun's disk [2]. Evidently thus the observed extraneous frequency decrease was accumulated only along the line of sight branch of the rays' path, which (as Earth-bound) was practically almost equipotential with respect to our Sun [2].

Similarly, also radio waves triggered by atomic clock, which was resting on the Earth's surface, showed then-unanticipated extraneous frequency decrease with increasing (practically almost equipotential) distance from their source [29]. Since the rays and waves were essentially grazing the respective surfaces of the Sun and Earth, those extraneous frequency decreases were evidently due to the nonradial angular (hence practically equipotential) effects alone [2].

Our Sun approached the path of the rays coming from Taurus A within a few solar radii. However, when line of sight of a certain pulsar approached within five degrees of our Sun, no evidence for any frequency decrease has been found [30], because the path of the rays' was too far away from the Sun in this particular case.

The equipotential rate of work done and thus also the field's potential energy spent on the work is tiny. It diminishes almost as fast as $1 / \mathrm{r}^{4}$ per unit volume. The frequency decrease becomes perceptible only on paths passing very close to the Sun's surface (i.e. near occultation). Yet the tiny nonradial effect causes over $25 \%$ latitude-dependent discrepancy in solar spectra taken from the Sun's limb (i.e. the apparent differential rotation of the Sun) [2]. It also retrodicts over $10.56 \%$ of the (observed in several independent experiments) excess over Einstein's prediction of deflection of light [2]. For in conjunction with enormous masses of planets and stars the impact of the tiny nonradial effects can become very significant indeed.

By Einstein's own admission his GTR was not devised for any other than just purely radial phenomena, for as he wrote: some tangential deviations [from the usual radial gravitational attraction] would be too slight if measured on the Earth [31], which was common assumption back then. Due to the deliberate Einstein's omission of any nonradial (i.e. tangential and binormal) gravitational effects, even his "flagship" prediction of deflection of light, which is evidently partly tangential phenomenon, was thus inaccurate. Several independent observers [32,33] have found formerly unexplained excess of 10-15\% [32] over the Einstein's prediction of deflection of light that originated from the reasonings that led him to his GTR.

The excess has been practically retrodicted by the new theory of nonradial effects of the usual radial gravitational force fields that also reconciled these two Sadeh experiments [2]. The theory of nonradial effects of gravity does not defy the (radial-only by design) GTR, but just complements it for purely (or just partly) angular nonradial phenomena (in radial/centerbound gravitational fields), whose theoretically foreseeable existence has been deliberately discarded by Einstein.

Yet when Sadeh has compared his experimental data, he found unexplained $390 \%$ discrepancy between the Sun-based and the Earth-based experiments. The discrepancy was impossible to reconcile back then in absence of nonradial effects. 
However, one can see that the discrepancy practically equals to the ratio of respective densities of matter of the Earth and Sun: $\mathrm{Q}_{\text {Earth }} / \mathrm{Q}_{\text {Sun }}=5.52 / 1.42 \cong 3.9$ see [34], which fact was first noticed and recognized as being due to their respective densities of matter in [2]. Since the nonradial effects are inversely proportional to exposure to density of matter [2], Sadeh's experimentally estimated coefficient for our Sun was 3.9 times larger than that for the Earth see [29] p.569. This was the simplest to discern experimental hint and evidence in favor of my claim that density of matter does matter, at least for nonradial angular and mixed (i.e. partly radial and nonradial) gravitational interactions at the present depth of inquiry [2].

Despite their exactness, the Sadeh experiments were notoriously ignored for $32+$ years and attempts trying to set foundation for their reconciliation rejected. Even when evidence is staring "in your face", incompletely defined concepts and misinterpretations of the Galilei and some other experiments stalled the progress.

Like specific gravity (i.e. density of matter), specific heat is also inversely proportional to work done by flow of the heat [35]. Evidently, whatever fills the space, be it heat or massmatter or charge, should (in some way) affect (at least) a certain class of physical phenomena happening within the space. Hence density of matter definitely counts for quasi-static angular nonradial effects acquired along equipotential surfaces within radial gravitational force fields of our Sun and Earth [2]. Unbeknownst to Sadeh, his experiments effectively proved Galilei wrong.

Actually only density of matter pertains to the material substance stored in mass-body, whereas mass (according to the Newton's formula F=ma) relates to its dynamics (force by acceleration). Only at relative rest both density of matter and of mass pertain to the amounts of material substance contained in massive bodies.

By replacing force by his curved spacetime, which is assigned like backpack to each single spacetime event [36,37], Einstein effectively geometrized physics of radial-only gravitational interactions. But because geometry is oblivious to any substance filling its spaces, density of matter did not matter in previous analyses.

Experimentally confirmed existence of nonradial effects of gravity did cast a shadow of doubt on validity of the tenuous Galilei's conclusion, but to challenge his claim responsibly required also demonstration that the nonradial effects are unavoidable and therefore their presence cannot be always neglected [4].

As pointed out by Kvasz, analytic philosophy of science considers science as a natural continuation of ordinary experience and common sense, which it is not; Galilei's attempt at mathematization of science was certainly hampered by lack of adequate mathematics, which was subsequently invented by others [38], but also by operationally deficient methodology, which still infuses and confuses physics.

\section{CONCLUSIONS}

The rate of work done or potential energy of radial/center-bound gravitational force fields can contain, in general, not only the usual radial component but also certain definitely nonradial subcomponents both linear and angular/equipotential.

In addition to dependence on masses and both radial- and perihelion distance, the (formerly ignored) angular nonradial subcomponents of potential energy that is spent on the work done by the field along equipotential surfaces also depend on the angular distance that is measured along equipotential surfaces surrounding the gravity center of the field's source mass that generates the locally dominant field. 
Moreover, angular nonradial subcomponents of the field's work done and its potential energy also depend (inversely though) on exposure (of the body/satellite that traverses the field) to (assumed as constant and uniformly distributed) density of matter of the field's dominant source mass, provided that the perihelion of the body's trajectory is very close to surface of the field's source mass.

Simply put: the work done by radial gravitational force field and thus also the corresponding to it expense of the field's potential energy is directly proportional to the local field's source mass and inversely proportional to density of matter of the field's source mass.

Although the equipotential rate of work done is very tiny, for it deeps almost as $1 / \mathrm{r}^{4}$ per unit volume, its operational and conceptual importance is enormous, because presence of the nonradial effects explains several formerly unanticipated curious phenomena revealed in experiments. Therefore, henceforth all previously coined force-field-based notions should be qualified as 'radial' in order for them (and also for theorems and conclusions derived from them) to remain valid.

Fairly detailed law for quasi-static nonradial angular gravitational effects in terms of work done and also of the corresponding to it potential energy has been synthesized upon the basis of operationally complete mathematical expression of rate of work done. The law implies that in addition to the regular attractive radial force (and also the, corresponding to it, usual radial gravitational frequency shift) a certain repulsive force field (and also the corresponding to it nonradial angular frequency decrease, i.e. angular gravitational redshift) arises from the nonradial angular (i.e. equipotential) subcomponents of an abstract differential-geometric representation of the usual radial/center-bound attractive gravitational force field.

Rigorous conceptual reanalysis of what was actually compared in two Sadeh experiments (even though not really measured in the sense of consciously taking it into account by Sadeh) showed that it was density of matter of the mass sources of the gravitational force fields of our Sun and Earth that were responsible for the (formerly unanticipated) frequency decreases in the rays coming from Taurus A, and also in the locally generated (on the Earth) radio waves, when the rays/waves traversed the gravitational fields surrounding the Sun and Earth, respectively.

If constitution/density of matter of the Sun and Earth matters for gravitational interactions, then constitution of matter of those small test bodies (which Galilei was dropping from the leaning tower of Pisa) should matter as well, even though its impact may be too minuscule to measure. After all, the Earth is just very huge amalgamated rock. If density of matter of the huge rock affects some gravitational interactions, so must also be true for those smaller rocks/objects too, for all rocks must be subject to the same universal laws of physics, no matter what their size.

These two experiments gave us thus affirmative answer to the question of whether constitution of massive bodies affects certain gravitational phenomena, definitely confirming that density of matter has detectable impact on the angular nonradial interactions that happen along the field's equipotential surfaces.

The formerly quite unanticipated frequency decreases discovered in these experiments have been explained and the experimental data reconciled by theory of nonradial effects of the usual radial gravity, which were routinely ignored due to the former, operationally incomplete definition of work done and of potential energy. Although the incomplete definition could still be tolerated for a single standalone force, it is not appropriate for central, radial/center-bound force fields. Possibility of presence of the nonradial effects was customarily neglected, mainly 
because of the mistaken Galilei's ad hoc conclusion that physical composition of material bodies allegedly did not affect gravitational phenomena.

The Galilei's claim that density of matter has no impact on gravitating bodies is definitely wrong. At the present depth of inquiry into classical gravitational interactions happening within attractive central, radial/center-bound gravitational force fields one can detect and measure (in appropriately designed and properly conducted unbiased experiments) the impact of (assumed as uniformly distributed and constant) matter density, which is acquired along equipotential surfaces i.e. in directions that are nonradial with respect to the gravity center of the main, locally dominant gravitational force field, provided perihelion of the satellite's trajectory path is located very close to the surface of the mass source of the local field.

\section{References}

[1] Jeffreys H., Jeffreys B., Methods of mathematical physics. Cambridge; Cambridge Univ. Press, 2001, p. 202.

[2] Czajko J., Chaos, Solit. Fract. 11 (2000) 2001-2016.

[3] Beiser A, Concepts of modern physics. New York: McGraw-Hill, 1973, p. $67 \mathrm{ff}$.

[4] Czajko J., Appl. Phys. Res. 3(1) (2011) 2-7.

[5] Czajko J., Stud. Math. Sci. 7(2) (2013) 40-54.

[6] Czajko J., Stud. Math. Sci. 7(2) (2013) 25-39.

[7] Duschek A., Vorlesungen über höhere Mathematik IV. Wien: Springer, 1961, pp. 229, 237.

[8] Sokolnikoff I. S., Sokolnikoff E. S., Higher mathematics for engineers and physicists. New York: McGraw-Hill, 1941, p. 218.

[9] Czajko J., Chaos Solit. Fract. 20 (2004) 683-700.

[10] Czajko J., Chaos, Solit. Fract. 11 (2000) 1983-1992.

[11] Thirring W., Problems of classical dynamical systems. CERN 75-8. Geneva: CERN, 1975, p.8ff.

[12] Czajko J., Chaos Solit. Fract. 19 (2004) 479-502.

[13] Bondi H., Eur. J. Phys. 14 (1993) 1-6.

[14] R. von Eötvös, Pekár D., Fekete E., Ann. Phys. (Leipzig) 68 (1922) 11-66.

[15] Ingard U., Kraushaar W. L., Introduction to mechanics, matter and waves. Reading, MA: Addison-Wesley, 1960, p. 40.

[16] Fischbach E., et al., Phys. Rev. Lett. 56 (1986) 3-6.

[17] Fischbach E., et al., Phys. Rev. Lett. 57 (1986) 1959.

[18] Baggott J., Farewell to reality. How fairytale physics betrays the search for scientific truth. London: Constable, 2013, p. 12 - compare also pp. 17, 20. 
[19] Campbell N. R., Mathematical Physics. [p.46-79 in: Weaver J. H. (Ed.) The World of Physics III. New York: Simon \& Schuster, 1987].

[20] Einstein A., Elementare Überlegungen zur Interpretation der Grundlagen der QuantenMechanik. [p. 33-40 in: Collective work: Scientific papers presented to Max Born. Edinburgh: Oliver \& Boyd, see p. 34].

[21] Aharonov Y., Bohm D., Phys. Rev. 115 (1959) 485-491.

[22] Trammel G. T., Phys. Rev. B134 (1964) 1183-1184.

[23] Peshkin M., Tonomura A., The Aharonov-Bohm effect. Part 1: Theory. Springer, Berlin, 1989, pp. 4ff, $20 \mathrm{ff}$.

[24] H. von Helmholtz, Vorlesungen über theoretische Physik I. Leipzig: Barth, (1903) p. 2.

[25] Melosh H. J., et al., Science 340 (2013) 1552-1555.

[26] Wheeler J. A., Time today. [pp. 21-50 in: Namiki M et al. (Eds.) Quantum physics, chaos theory and cosmology. Woodbury, NY: AIP Press, 1996].

[27] Szekeres G., Nature 220 (1968) 1116-1168.

[28] Sadeh D., Knowles S. H., Yaplee B. S., Science 159 (1968) 307-308.

[29] Sadeh D., Knowles S., Au B., Science 161 (1968) 567-569.

[30] Sadeh D., Hollinger J. P., Knowles S. H., Science 162 (1968) 897-898.

[31] Einstein A., The Foundations of the General Theory of Relativity [pp. 111-164 in: H.A. Lorentz et al. The principle of relativity. New York: Dover, 1923, see Einstein's disclaimer on p. 161].

[32] Merat P., Astron. Astrophys. 32 (1974) 471-475.

[33] Dyson F., Nature 106 (1921) 786-787.

[34] Weast R. C. (Ed.) Handbook of Chemistry and Physics. 51st ed. Cleveland, OH: The Chemical Rubber Co., 1970, p. F145.

[35] Kellog O. D., Foundations of potential theory. Berlin: Springer, 1929, p. $77 \mathrm{f}$.

[36] Geroch R., General relativity from A to B. Chicago: The Univ. of Chicago Press, 1978, pp. 166, 171.

[37] O’Neill B, Semi-Riemannian geometry with applications to relativity. New York: Academic Press, 1983, p. 171.

[38] Kvasz L., Acta Phys. Slov. 62(6) (2012) 519-614; see pp. 522, 524. 